CARNETS DE Carnets de géographes

GÉOGRAPHES.

$6 \mid 2013$

Géographie des faits religieux

\title{
Renouveau des campagnes françaises
}

Évolutions démographiques, dynamiques spatiales et recompositions sociales

\section{Pierre Pistre}

\section{OpenEdition}

1 Journals

Édition électronique

URL : https://journals.openedition.org/cdg/963

DOI : $10.4000 / c d g .963$

ISSN : 2107-7266

Éditeur

UMR 245 - CESSMA

Référence électronique

Pierre Pistre, «Renouveau des campagnes françaises », Carnets de géographes [En ligne], 6| 2013, mis en ligne le 01 septembre 2013, consulté le 15 février 2023. URL : http://journals.openedition.org/cdg/ 963 ; DOI : https://doi.org/10.4000/cdg.963

Ce document a été généré automatiquement le 29 septembre 2020.

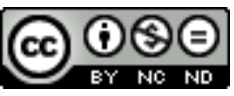

Creative Commons - Attribution - Pas d'Utilisation Commerciale - Pas de Modification 4.0 International - CC BY-NC-ND 4.0

https://creativecommons.org/licenses/by-nc-nd/4.0/ 


\title{
Renouveau des campagnes françaises
}

\author{
Évolutions démographiques, dynamiques spatiales et recompositions \\ sociales
}

Pierre Pistre

1 Cette thèse présente une analyse détaillée du renouveau démographique des campagnes françaises amorcé dans les années 1970-1980. Elle met l'accent sur les processus qui ont conduit à des évolutions de population de plus en plus positives, et elle accorde une attention centrale aux individus et groupes venus s'installer en France rurale. Elle propose ainsi un regard actualisé sur ce que Bernard Kayser (1989) a pu appeler la renaissance rurale, quand d'autres ont parlé de reprise (Thomsin, 2000 ; Cognard, 2001), de revitalisation (Rouzier, 1990 ; Font, 1994; Chevalier, 2003) ou de repeuplement (Thomsin, 2001 ; Hirczak et al., 2011) des campagnes françaises.

2 Rarement appréhendé comme un objet de recherche spécifique, le renouveau démographique rural est analysé dans ses caractéristiques spatiales, ses variations temporelles et ses composantes sociodémographiques. L'étude relève de la géographie humaine, de la géographie rurale et de la géographie de la population; elle se veut également interdisciplinaire, à la croisée de la géographie, de la démographie, de la sociologie et du champ des études rurales. Par ailleurs, il s'est agi de diversifier les démarches d'analyse des croissances démographiques en France rurale ; l'association de méthodes quantitatives (statistiques univariées, bivariées, multivariées) et qualitatives (observation, entretiens semi-directifs) a notamment permis d'examiner des tendances au renouveau rural à plusieurs échelles géographiques : locale, régionale et nationale. 


\section{De la diversité du renouveau démographique des campagnes françaises}

3 Le cœur problématique de cette thèse se situe dans la diversité intrinsèque - supposée puis constatée - du renouveau démographique contemporain des campagnes françaises. Celui-ci a fortement évolué depuis ses prémices dans les années 1970-1980. Il est pluriel par les processus et les populations impliqués. Il reste également contrasté selon les régions et les territoires, appelant des analyses de différenciation spatiale du phénomène. La mise en évidence d'une variété de tendances et de facteurs explicatifs a été ainsi privilégiée.

Plusieurs hypothèses de travail ont été avancées : d'ordre spatial, démographique et sociologique. Au regard des décennies passées, le renouveau rural des années 2000 se caractériserait par une diffusion bien au-delà de la proche influence urbaine. La poursuite et le développement du phénomène s'expliqueraient parallèlement par des installations résidentielles toujours plus nombreuses et diffuses en France rurale. L'accentuation du renouveau résulterait également de la diversification du profil des nouveaux résidents plus que de l'accroissement de l'attrait des campagnes pour certaines populations restreintes. En définitive, les analyses présentées dans la première partie de la thèse, intitulée Dynamiques démographiques et migratoires en France rurale, avalisent globalement ces hypothèses générales; il est malgré tout difficile d'affirmer que la diversification du profil des nouvelles populations a été le principal facteur de la diffusion et de l'accentuation du renouveau rural.

Les deuxième et troisième parties, intitulées Processus de gentrification et renouveaux des campagnes françaises et Populations retraitées et renouveaux des campagnes françaises, ont vocation d'approfondissement des évolutions sociodémographiques en France rurale. Du fait des dynamiques migratoires et de logement qui sont inhérentes aux processus de gentrification (dont rurale), et de la propension des populations retraitées à s'installer dans les campagnes françaises, il s'agissait de les considérer comme des composantes centrales du renouveau rural contemporain. Les résultats obtenus sur ces deux axes de travail sont plus nuancés que pour les premières hypothèses générales. Gentrification rurale et populations retraitées ont participé indéniablement aux reprises démographiques observées, mais en étant loin d'épuiser les facteurs explicatifs du phénomène, et notamment de son développement au cours des années 2000.

\section{Du national au local, quantifications statistiques et exemplifications qualitatives}

6 La méthodologie de travail repose sur deux volets principaux; le premier correspond aux traitements quantitatifs effectués à l'échelle nationale. Une grille de définition des campagnes françaises a tout d'abord été réalisée par classification statistique des bassins de vie métropolitains (Carte 1). Cette typologie distingue trois grands types de campagnes (des villes, productives, résidentielles); utilisée comme nomenclature de référence, elle a permis de mettre en évidence des différences significatives, de dynamiques démographiques, migratoires ou de logement, parmi les campagnes françaises. 


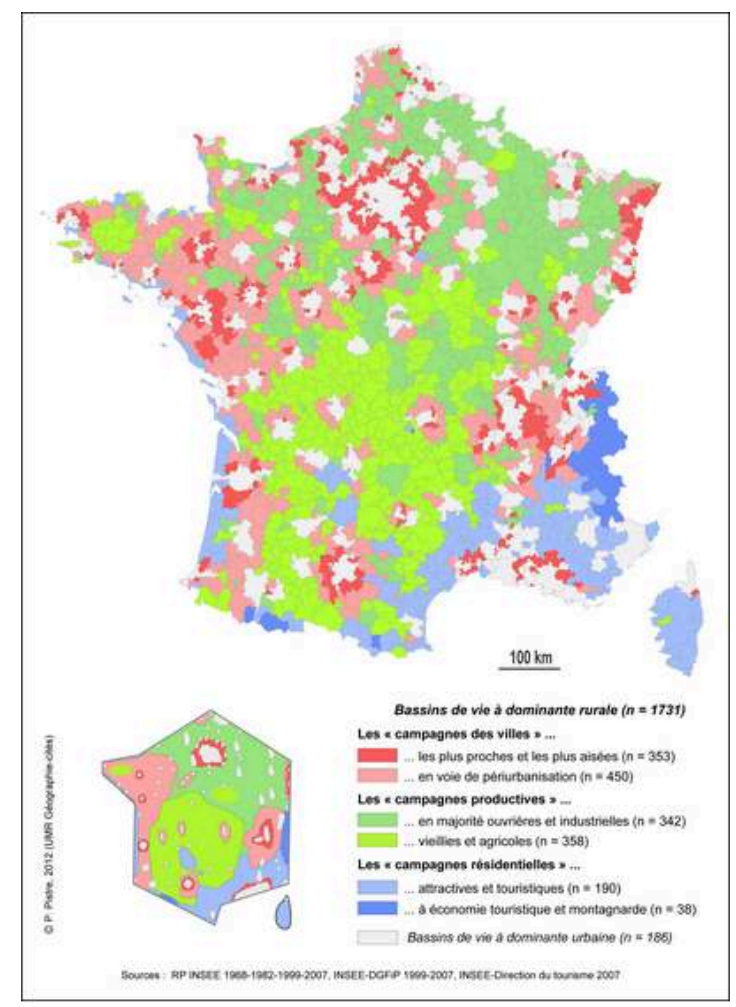

7 Pour satisfaire ensuite aux objectifs de quantification et d'interprétation nationale du renouveau rural, des sources statistiques variées ont été utilisées : les fichiers détail du recensement INSEE de la population depuis 1968, les enquêtes Logement INSEE de 1992 à 2006, l'enquête Histoire de vie INSEE/INED de 2003, et encore des données de la Direction du tourisme sur les populations présentes et les hébergements touristiques. Cet ensemble de bases de données a été notamment mobilisé pour détailler l'analyse des migrations et mobilités résidentielles vers les campagnes françaises: selon les origines et les destinations, les distances parcourues, les âges et les catégories socioprofessionnelles, la composition des ménages, les trajectoires résidentielles, les nationalités ou les lieux de naissance.

Le deuxième volet méthodologique correspond à trois études de territoire réalisées entre 2010 et 2012, en Haute-Provence, aux confins sud-ouest de la Haute-Vienne, et dans le Haut- Languedoc (Carte 2). Elles ont reposé sur l'association de trois formes d'investigation. Des analyses statistiques exploratoires ont aidé à la sélection et à la définition des terrains d'étude, puis à examiner le contenu et la diversité des formes locales de renouveau rural. Des observations ont permis de s'imprégner des territoires étudiés, de leur organisation spatiale, de leur occupation démographique et de signes multiples d'évolution. Enfin, des entretiens semi- directifs ont été menés auprès d'acteurs locaux, de personnes ressources, de professionnels du marché immobilier et de nouveaux résidents surtout retraités; ceux-ci ont été d'une aide précieuse pour contextualiser, expliciter et exemplifier l'observable et les résultats statistiques.

Carte 2. Localisation des terrains d'étude dans la moitié sud de la France 


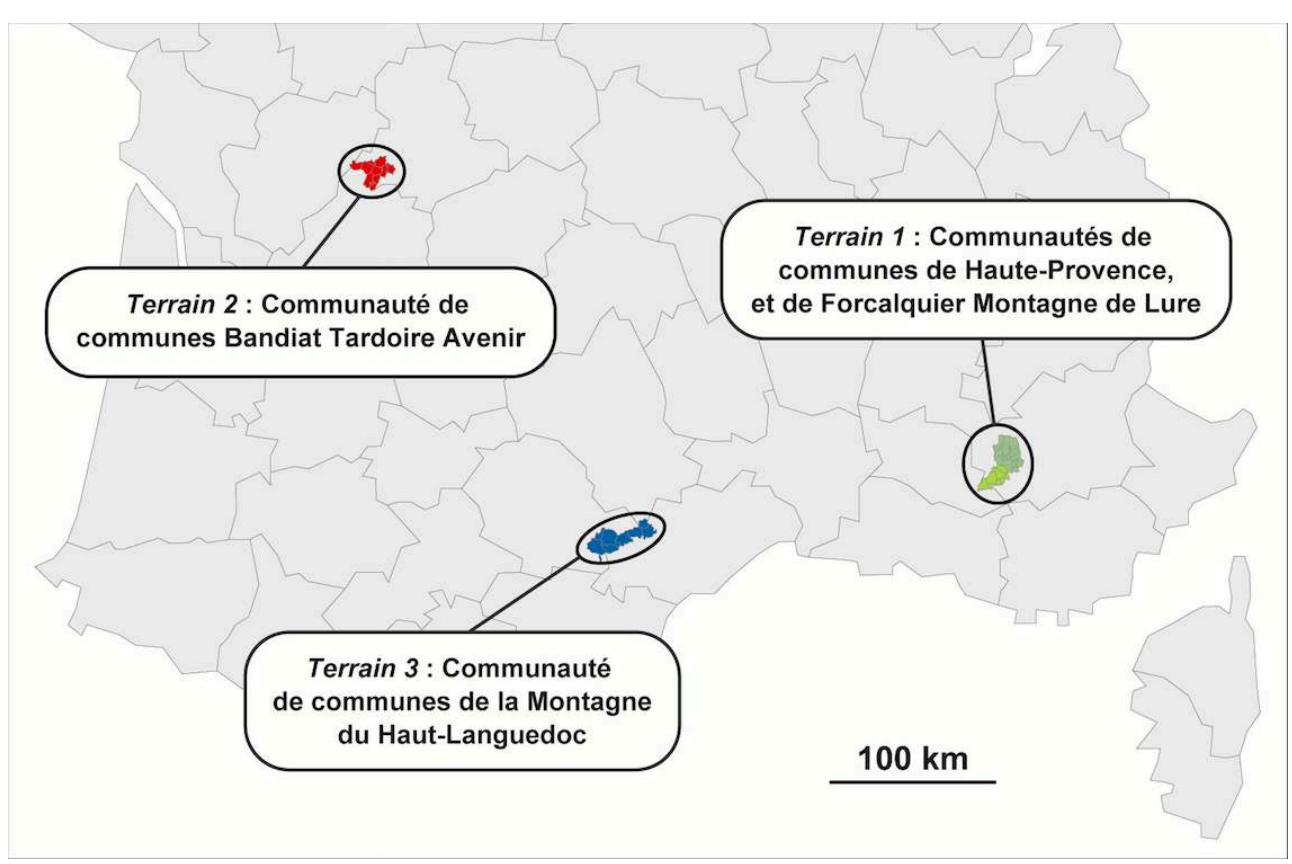

Les campagnes françaises ont bien connu des reprises démographiques de plus en plus significatives et généralisées depuis les années 1970-1980. La progression de l'attractivité résidentielle a été le principal moteur des croissances rurales, en particulier pour les campagnes productives et résidentielles. En outre, des processus variés - de périurbanisation, de rurbanisation, de néoruralisation, de gentrification, de développement résidentiel et touristique... - ont contribué à cet état de fait, et chacun d'eux a plus imprégné un large éventail de territoires qu'il n'a été exclusif pour telle ou telle campagne. Ainsi, le renouveau démographique contemporain des campagnes françaises s'explique en premier lieu par la concomitance de ces processus, à l'échelle nationale et de la plupart des territoires locaux.

\section{BIBLIOGRAPHIE}

Chevalier P. (2003), « Les potentialités des différents espaces ruraux dans une perspective de revitalisation », Revue de l'Economie Méridionale, n² 204, pp. 511-524.

Cognard F. (2001), « Reprise démographique et nouvelles populations dans les moyennes montagnes françaises », Espace, populations, sociétés, Vol 19, nº 1-2, pp. 53-58.

Font E. (1994), « Un schéma d'évolution démographique pour les communes rurales », Revue de l'Economie Méridionale, n 165-166, pp. 27-47.

Hirczak M., Chevalier P., Dedeire M., Razafimahefa L. (2011), « Dynamiques rurales et trajectoires démographiques : comparaison France, Italie, Espagne », L’information géographique, Vol 75, $n^{\circ} 2$, pp. 68-87.

Kayser B. (1989), La renaissance rurale : sociologie des campagnes occidentales, Armand Colin, Paris, 316 p. 
Rouzier J. (1990), « La mutation de l'arrière-pays méditerranéen ou un modèle pour la revitalisation des communes rurales ", Revue d'Economie Régionale et Urbaine, n 5, pp. 695-713.

Thomsin L. (2000), « La reprise démographique rurale en Wallonie et Europe du Nord-Ouest», Espace, populations, sociétés, Vol 18, $\mathrm{n}^{\circ} 1, \mathrm{pp} .83-99$.

Thomsin L. (2001), « Repopulation et mobilités rurales », Espaces, populations, sociétés, Vol 19, n $1-2$, pp. 13- 17.

\section{Lien électronique de la thèse}

http://halshs.archives-ouvertes.fr/tel-00764869/

Discipline

Géographie

Directrice

Catherine Rhein

\section{Université}

Université Paris-Diderot

Membres du jury de thèse, soutenue le 7 décembre 2012

Catherine Bonvalet, Directrice de recherche, INED (rapporteur)

Henry Buller, Professeur, Université d'Exeter (UK) (rapporteur)

France Guérin-Pace, Directrice de recherche, INED (présidente du jury)

Olivier Orain, Chargé de recherche, CNRS (examinateur)

Catherine Rhein, Directrice de recherche, CNRS (directrice de thèse)

Frédéric Richard, Maître de conférences, Université de Limoges (examinateur)

Situation professionnelle à l'issue de la thèse

ATER à l'Université Paris Diderot (Paris 7)

Membre associé à l'UMR8504 - Géographie-cités

Contact de l'auteur

pierre.pistre@parisgeo.cnrs.fr

INDEX

Thèmes : Carnets de soutenances

Mots-clés : campagne, France 